\title{
Imaging Plasmon Modes in Metallic Nanostructures with Correlated Optical and Electron Microscopy
}

Vighter O. Iberi*, Shuzhou Li**, Donovan Leonard***, Stephen J. Pennycook***, Beth S. Guiton***,**** Jon P. Camden*

* Department of Chemistry, University of Tennessee, Knoxville, TN 37996-1600

** School of Materials Science \& Engineering, Nanyang Technological University, Singapore

*** Materials Science \& Technology Division, Oak Ridge National Laboratory P.O. Box 2008, Oak Ridge, TN 37831

**** Department of Chemistry, University of Kentucky, Lexington, KY 40506-0055

The primary goal of this project is to correlate the optical measurements of nanostructures generated by the surface plasmons using Resonant Rayleigh Scattering and STEM/VEELS. Additionally, we are studying shape effects because it is believed that the different modes that are observed in plasmon resonances depend on the shape of the nanoparticles [1].The study of plasmons is at the core of Surface-Enhanced Raman Spectroscopy (SERS). Surface plasmons are generated from the interaction of light with the electrons on the surface of a metal and are responsible for the electromagnetic enhancement mechanism in SERS.

Briefly, colloidal silver nanoparticles (nanoComposix, San Diego, CA) were used without further purification. Our experiments utilized two types of TEM grids: (1) a copper grid coated with 2-3 nm of amorphous carbon and formvar (Ted Pella Inc. \#822-01118) and (2) $20 \mathrm{~nm}$ thick $\mathrm{SiN}_{\mathrm{x}}$ membrane supported on silicon (SPI supplies \#4105SN). These two grid types allowed comparison of STEM/EELS measurements on both conducting and non-conducting substrates. A $2 \mu \mathrm{L}$ aliquot of the colloidal sample was drop coated directly onto a TEM membrane support and the solvent was allowed to evaporate. Resonance-Rayleigh scattering measurements were performed using an inverted microscope (Nikon, Ti-U) equipped with a dark field condenser. Scattered light from an individual nanoparticle was collected with $100 \mathrm{X}$ (Nikon, $0.7<\mathrm{NA}<1.4$, oil immersion) objective and coupled into a dispersive imaging spectrometer. Light was detected on a liquid nitrogen-cooled back-illuminated charge couple detector (CCD) and the dark-field scattering spectrum of each individual nanoparticle was obtained. A wide-field image of the silver nanoparticles on TEM grid was also recorded to serve as a map for subsequent location in the STEM. This method enables correlated optical and STEM measurements from the exact same nanostructures. The STEM EELS spectrum imaging (SI) measurement was performed on a VG Microscopes HB501UX STEM with Nion aberration corrector and Gatan Enfina EEL spectrometer. In each case the rod in question was found by comparison of the optical map to the pattern of particles imaged in the STEM at very low resolution; after identification, a higher resolution annular dark field (ADF) image was collected, followed by the SI. The spectrometer dispersion was set to $0.05 \mathrm{eV}$ per channel with an exposure time of $0.05 \mathrm{~s}$ per spectrum. The pixel size/density was chosen to give a total acquisition time of around 15 minutes for a single SI. The energy resolution (as measured by the FWHM of the zero loss peak) was $\sim 0.45-0.50 \mathrm{eV}$. Principal component analysis (PCA) was used to extract the experimentally measured modes from the SI.

In Figure 1. the left image shows the presence of modes that are excited leading to the observed resonant Rayleigh scattering spectrum. Figure 2. shows the transverse plasmon mode (experiment and theory) of the exact same Ag nanorod at a particular energy $(1.65 \mathrm{eV})$. Other modes (not shown) such as longitudinal and multipolar modes were also excited. The success of this project will provide new tools to study plasmons important in Single Molecule Surface- 
Enhanced Raman Spectroscopy (SMSERS), bio/chemical sensing [2-4], and other forms of surface-enhanced spectroscopy. [5]

\section{References}

[1] Y. Wang, et al., Ultramicroscopy (109), 2009, pp. 1110-1113.

[2] R. Elghanian, et al., Science 277 (5329) (1997) 1078-1081.

[3] G. Bauer, F. Pittner, T. Schalkhammer, Mikrochimica Acta 131 (1-2) (1999) 107-114.

[4] A.J. Haes, et al., Journal of Physical Chemistry B 108(22) (2004) 6961-6968.

[5] Research sponsored by the DOE Office of Basic Energy Sciences, Materials Sciences and Engineering Division (DNL, SJP).
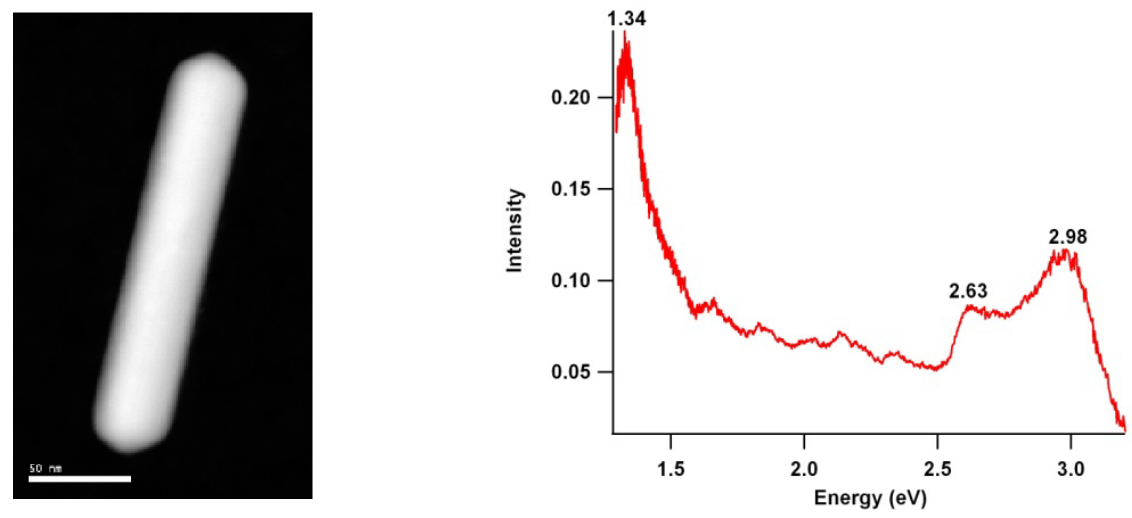

angle annular dark field (HAADF) image of a nanorod 202nm x 40nm(left) and its corresponding resonance Rayleigh scattering spectrum (right).
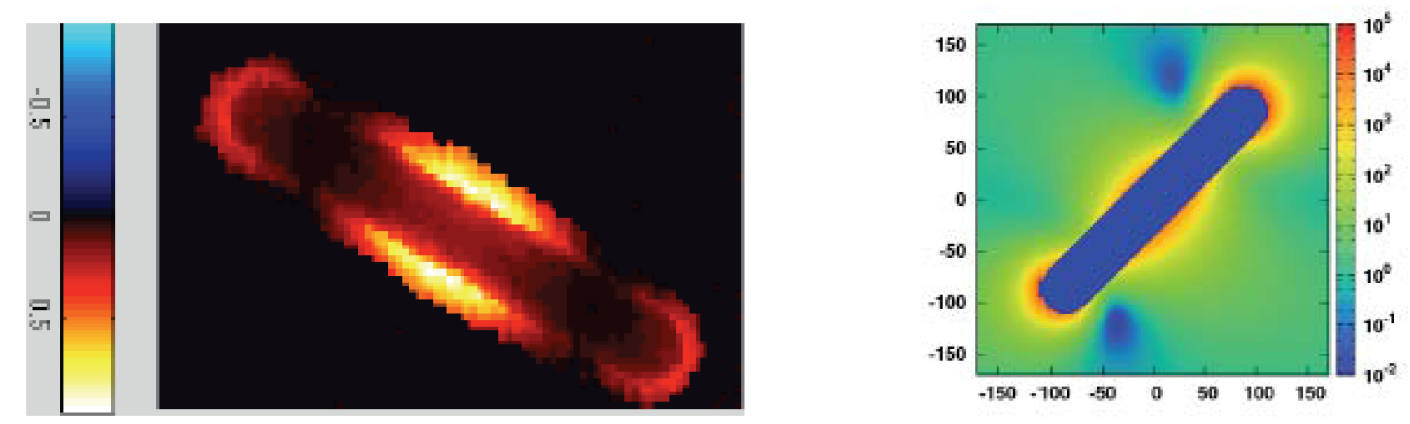

FIG. 2. Experimental EELS mapped plasmon mode of the exact same Ag nanorod at $1.65 \mathrm{eV}$ (right) and its corresponding discrete dipole approximation calculation at $1.7 \mathrm{eV}$ (left) 